\title{
Philippe Subra, Géopolitique locale. Territoires, acteurs, conflits
}

Paris, Armand Colin, 2016, 336 p.

Mark Bailoni

\section{OpenEdition}

\section{Journals}

Electronic version

URL: http://journals.openedition.org/rge/5945

DOI: $10.4000 /$ rge.5945

ISSN: 2108-6478

\section{Publisher}

Association des géographes de l'Est

Printed version

Date of publication: 1 December 2016

ISSN: 0035-3213

\section{Electronic reference}

Mark Bailoni, «Philippe Subra, Géopolitique locale. Territoires, acteurs, conflits », Revue Géographique de l'Est [Online], vol. 56 / n³-4 | 2016, Online since 29 December 2016, connection on 23 September 2020. URL : http://journals.openedition.org/rge/5945 ; DOI : https://doi.org/10.4000/rge.5945

This text was automatically generated on 23 September 2020 .

Tous droits réservés 


\section{Philippe Subra, Géopolitique locale. Territoires, acteurs, conflits}

Paris, Armand Colin, 2016, 336 p.

Mark Bailoni

1 Cet ouvrage, richement illustré par des cartes et de nombreux schémas clairs et efficaces, est un manuel indispensable à tout étudiant se destinant aux métiers de l'aménagement $d u$ territoire, à tout professionnel du secteur, voire à tout citoyen. Philippe Subra apporte en effet toutes les clés pour comprendre et décrypter les jeux d'acteurs et les enjeux de pouvoir au niveau local en France, sources de conflits véritablement géopolitiques. Ce manuel est d'autant plus essentiel que les questions abordées ici sont au cœur de l'actualité et suscitent de nombreux débats dans la société française.

2 La longue introduction de l'ouvrage explique les fondements et l'intérêt d'une

\section{GÉOPOLITIQUE} LOCALE

\author{
Territoires, acteurs, conflits
}

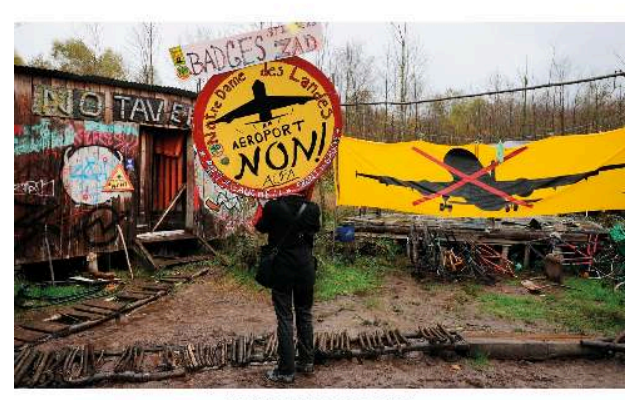

ARMAND COLIN approche de géopolitique locale. Le travail de Philippe Subra s'inscrit dans la démarche entamée par Yves Lacoste, Béatrice Giblin et l'équipe de la revue Hérodote. En effet, si l'usage médiatique du terme géopolitique renvoie à des grandes questions internationales, à des guerres ou à des tensions entre Etats, armées rebelles et groupes terroristes, Yves Lacoste a publié dès les années 1980 une Géopolitique des régions françaises, ouvrant la voie à l'usage d'une démarche géopolitique pour analyser des questions internes aux Etats et aux sociétés démocratiques. Il a poursuivi ce travail 
dans les années 1990, analysant les enjeux géopolitiques auxquels était confrontée la nation française - tout comme dans le récent ouvrage co-écrit avec Frédéric Encel, Géopolitique de la nation France (PUF, 2016). Béatrice Giblin a également fortement contribué à l'élargissement des champs d'études de la géopolitique, par un travail d'analyse des rapports de force et des stratégies d'élus et de partis politiques, ou des enjeux identitaires dans certains territoires (« nationalismes régionaux » en France, les " banlieues " dans le Dictionnaire des banlieues notamment). Philippe Subra a également contribué à ce travail par son analyse des jeux d'acteurs dans le Valenciennois (1996), dans la "Banlieue rouge » ou dans l'élaboration du projet de Grand Paris (2012). Il a aussi développé une véritable approche de géopolitique de l'aménagement du territoire, faisant l'objet d'un autre ouvrage chez Armand Colin (2007, réédité en 2014).

3 La géopolitique locale étudie ainsi les conflits liés à l'aménagement du territoire, entre partisans de la construction de tel équipement ou de tel lotissement, et opposants (écologistes, agriculteur exproprié, opposant politique, riverain craignant les nuisances, simple citoyen, etc.), la question plus générale de la gouvernance territoriale et les relations entre les différentes strates de pouvoir du "millefeuille administratif " français, ainsi que la question du contrôle local ou régional du pouvoir. Philippe Subra précise dans les conflits locaux qu'il étudie ici, « il n'y a pas de morts, sauf exception. Pas d'envoyés spéciaux de CNN, mais un article dans Ouest-France ou dans Le Provençal relatant une réunion et un débat public un peu mouvementés ou un sujet de trois minutes dans l'édition régionale du journal télévisé de France 3 montrant les images d'une manifestation. (...) Pourtant, il s'agit bien (...) de conflits géopolitiques car leur enjeu, fondamentalement, est l'usage, donc le contrôle de territoires. La géopolitique locale peut donc être vue comme une sorte de " géopolitique soft ", infiniment moins violente et moins médiatisée que l'autre, celle des conflits internationaux et des guerres civiles, mais qui n'est pas moins géopolitique car elle porte, elle aussi, pour reprendre la définition qu'Yves Lacoste a donnée de la géopolitique, sur des rivalités de pouvoir sur du ou des territoires. » (pp.7-8)

4 Pour décrypter ces conflits locaux et ces rivalités politiques, Philippe Subra montre l'importance d'analyser la nature des acteurs en rivalité, la logique et les intérêts défendus par ces acteurs, les territoires dont ils convoitent ou défendent le contrôle ou l'usage, et leurs représentations géopolitiques (Chapitre 1). On retrouve ici les fondements d'une démarche géopolitique classique, définie et élaborée par Yves Lacoste et l'équipe d'Hérodote, et utilisée pour analyser d'autres types de conflits.

Cet ouvrage est ensuite structuré en quatre parties, subdivisées en onze chapitres. Plusieurs études de cas complètent et illustrent les chapitres. La première partie analyse les mécanismes des conflits d'aménagement, nés de logiques différentes. Philippe Subra distingue en effet trois grandes logiques conflictuelles : la défense d'un équipement, d'un service ou d'une usine menacé de fermeture ; la compétition entre des territoires pour attirer un équipement ou un grand évènement ; et la contestation de projets d'aménagement du territoire considérés comme nuisibles (pp.66-68). Si parfois les trois logiques peuvent coexister dans un même conflit d'aménagement, autour du même projet, c'est surtout la logique de l'aménagement rejeté qui provoque la plus forte et la plus large mobilisation, et donc la plus importante couverture médiatique. Ces conflits se sont généralisés en France depuis les années 1970, et, pour certains d'entre eux, sont devenus des questions politiques nationales (projets 
d'extension d'un camp militaire au Larzac et d'une centrale nucléaire à Plogoff dans les années 1970, projets d'aéroport à Notre-Dame-des-Landes ou de barrage à Sivens aujourd'hui). Leurs enjeux dépassent ainsi parfois ceux de l'aménagement du territoire. Philippe Subra explique les raisons de cette généralisation : contexte économique et social, préoccupations environnementales croissantes, rapports au territoire, méfiance à l'égard des " élites ", de l'Etat, des hauts-fonctionnaires et même des élus locaux (pp. 59-61).

6 L'auteur applique une démarche géopolitique classique à l'analyse de ces conflits, prouvant toute sa pertinence ici : l'étude du rapport de force entre acteurs, la description des acteurs et de leurs motivations (comme le phénomène " Nimby », pp. 68-70, et par extension, le très pertinent concept de "Nimby social " pp.58-59), l'approche critique des représentations contradictoires qui sont évoquées dans les discours (" intérêt général ", " nature ", " désertification ", " grand projet inutile », etc. : pp.74-81), mais aussi l'analyse multiscalaire qui prend tout son sens dans l'étude des « ZAD » et des réseaux de la contestation (pp.85-103). Les études de cas de Notre-Damedes-Landes et de Sivens mettent en application cette approche géopolitique et montrent son intérêt, à partir de ces deux exemples emblématiques et incontournables de contestation de grands projets d'aménagement. Le travail cartographique sur l'évolution de la ZAD de Sivens (p.102), réalisé à partir de six croquis rappelant les études stratégiques et tactiques des grandes batailles, est une très belle illustration de ces rivalités locales entre zadistes, agriculteurs et autorités pour le contrôle du territoire.

$7 \quad$ La deuxième partie décrit les tactiques employées pour sortir d'une situation de conflit : la concertation, le débat public et même le référendum local. C'est la partie la plus technique de l'ouvrage : Philippe Subra revient sur les fondements législatifs qui ont amené à l'organisation des débats publics et à leurs évolutions, et explique leur fonctionnement (pp.113-142). Il dresse ensuite le bilan des 86 débats publics menés entre 1997 et fin 2015 en France. Il montre leurs impacts - parfois très mesurés - sur la conflictualité et la situation de tension, et leurs effets concrets sur les projets, créant une " échelle de Richter de l'effet débat public sur les projets ", allant du niveau 1 " validation pure et simple » au niveau 7 « abandon définitif du projet » (pp.161-163).

La troisième partie aborde la question de la gouvernance territoriale en France et de ses enjeux géopolitique. Philippe Subra revient tout d'abord sur les origines du découpage territorial du territoire français, qui a abouti à ce qui est souvent qualifié de « millefeuille territorial» (p.171). Il explique les contextes géopolitiques contemporains à la création des départements, puis à leur remise en cause, aux premiers projets de régionalisation, à la création effective des régions puis à leur redécoupage. L'auteur analyse aussi les différentes phases de la décentralisation, de la réforme de 1982 aux lois les plus récentes (comme la loi NOTRe de 2015) et leurs conséquences géopolitiques locales. Il explique aussi les différentes phases de ce qu'il appelle " la révolution intercommunale ", des origines (premiers dispositifs de la fin du XIXe) à la création des métropoles (loi MAPTAM de 2014). Philippe Subra montre le caractère très géopolitique de ces différentes phases de décentralisation et de réformes territoriales, quel que soit le niveau concerné, de la commune à la région. Il montre clairement que derrière ces réformes, on trouve un jeu d'acteurs complexe dans lequel s'affrontent différents niveaux de pouvoirs (de l'Etat à la commune), différents lobbies d'élus et de hauts fonctionnaires, différents intérêts partisans ou différentes personnalités politiques, qui 
tous entendent défendre leurs pouvoirs dans les transferts de compétences, dans la création de nouveaux territoires politiques (régions, intercommunalités rurales, Grand Paris, métropoles) ou dans leur redécoupage (par exemple, question de la suppression des départements ou regroupement des régions). Les cas du Grand Paris et de la métropole lyonnaise montrent très bien ces jeux d'acteurs entre élus locaux, départementaux, régionaux et nationaux. Cette partie s'achève par l'étude de ces questions de gouvernance territoriale en Allemagne, Espagne, Belgique, Italie et Royaume-Uni. Si cette approche comparative pourrait être entièrement justifiée et très éclairante, elle apparait trop superficielle, car les cas de ces pays ne sont qu'à peine développés. Certes, il ne s'agit pas là du propos central du livre : pour être exhaustive, cette comparaison mériterait - au moins - un ouvrage en entier !

9 La quatrième et dernière partie s'intéresse à la géopolitique électorale au niveau local. Philippe Subra analyse ce qu'il appelle les " systèmes géopolitiques locaux ", qui caractérisent certains territoires dominés massivement ou exclusivement par un acteur politique (personnalité élue, collectif d'élus ou parti politique). Il explique comment ces responsables politiques contrôlent réellement leur territoire et assure leurs réélections. Comme il le montre bien, les cas de "la banlieue rouge ", des Hauts-deSeine et de Marseille et des Bouches-du-Rhône sont de parfaits exemples de systèmes géopolitiques locaux, dont le cadre dépasse largement le strict cadre politique pour englober aussi des organismes publics (offices HLM par exemple), des syndicats, des entreprises partenaires ou des associations locales. Là encore, l'approche géopolitique permet d'analyser ces véritables jeux d'acteurs et ces rivalités de pouvoirs sur des territoires. S'ils assurent une forme de pérennité politique sur les territoires concernés, ces systèmes géopolitiques locaux ne sont pas pour autant tout à fait figés, et ils peuvent être influencés par les évolutions des rapports de force au niveau local, que Philippe Subra analyse dans son dernier chapitre.

Cet ouvrage offre ainsi au lecteur un panorama à la fois concis et complet des grands enjeux de la géopolitique locale en France. Il permet au (futur) professionnel de l'aménagement du territoire d'analyser et de mieux appréhender un certain nombre de problématiques auxquelles il sera confronté au cours de sa carrière, comme la contestation inévitable des projets et de certaines décisions ou les jeux de pouvoirs et d'influences mêlant élus de tous niveaux et autres acteurs locaux. Il permet à un public plus large, initié ou non, de mieux comprendre les enjeux véritablement géopolitiques et de mieux décrypter certains discours politiques dans de grandes questions au cœur de l'actualité nationale. Cet ouvrage contribue également à montrer que le raisonnement géopolitique s'applique certes à l'analyse des grandes questions internationales, mais aussi à celle des jeux d'acteurs et de pouvoirs locaux auxquels sont directement confrontés les citoyens. 


\section{AUTHOR}

\section{MARK BAILONI}

LOTERR, Université de Lorraine, 23, boulevard Albert Ier, 54000 NANCY, mark.bailoni@univlorraine.fr 